\title{
Transforming Computer Science Educators Landscape Using the Greek School Network
}

\author{
http://dx.doi.org/10.3991/ijac.v6i2.2779 \\ M. Paraskevas and T. Zarouchas \\ Computer Technology Institute and Press “Diophantus”, Patras, Greece
}

\begin{abstract}
This study presents the potential use of Greek School Network (GSN) in order to provide a robust and versatile e-learning course for computer science educators, allowing the exploitation of advanced IT services and establishing a modern and attractive education environment in the Greek Society. A time Scheduling and Tele-Education Platform (t-STEP) is introduced that compensates the growing needs on information and communication technologies and provides the appropriate training framework to computer science educators to excel their technical proficiency.
\end{abstract}

Index Terms-computer science educators, e-learning, Greek School Network, training

\section{INTRODUCTION}

The Ministry of Education (MoE, http://www.minedu.gov.gr) has established the strategic plan for the "Digital School", in order to modernize fundamental education processes through the broad use of the information and computer technologies (ICTs). In the context of the "Digital School", on-going and near future actions, in both primary and secondary education, evolve the wide use of innovative technologies, in order to enhance the traditional and lecture-dominant teaching methods and provide a flexible and attractive learning environment. Related actions include the following:

- Initiation of "Bring You Own Device" (BYOD) methodology [1] to primary and secondary schools

- Installation of mobile computer laboratories with enhanced functionalities (i.e., broadband wireless access, multimedia equipment) and integration of advanced devices such as interactive whiteboards

- Schools interconnection to municipal optical metropolitan area networks, enabling high-speed optical access to GSN

- Interactive and experimental learning environment considering the use of (a) tablets or e-book readers, (b) low cost electronic prototyping platforms (i.e., Arduino kit, Raspberry Pi), (c) 3D printers and (d) programmable robotic and artificial intelligent systems

- $3 \mathrm{D}$ visualization and interaction [2]

In order the above activities achieve their potential goals, an updated and targeted training course should be provided to computer science educators. This will establish a flexible framework that will promote the utilization of information and communication technology infrastructure in both primary and secondary schools. The e-learning course will be realized using an appropriate designed time Scheduling and Tele-Education Platform ( $\mathrm{t}-$ STEP) that exploits the functionality of the Greek School Network (GSN) [3] which delivers high quality network and telematics services to its end users [3-5].

This paper is organized as follows: Section II introduces the Greek School Network (GSN). Section III describes the basic scheme and functionality of the proposed t-STEP. Section IV analyzes the conceptual framework of the proposed training course while its complementary adaptation framework is presented in Section V. Finally, conclusions are drawn in Section VI.

\section{BRIEF OVERVIEW OF GREEK SCHOOL NETWORK}

The Greek School Network (GSN, http://www.sch.gr) was founded in 1998 and constitutes the educational intranet of the Ministry of Education [3]. GSN interconnects all Greek schools, educational administration offices and organizations and also provides advanced e-services to students, teachers, and school administrator communities [4]. It was funded by the Community Support Frameworks of the European Union and is currently maintained and supported by a group of twelve research institutes and universities, under the supervision of Computer Technology Institute and Press "Diophantus" (CTIP, http://www.cti.gr) [5]. It is the biggest national public network according to the number of users, and is recognized internationally as a remarkable educational network that promotes the introduction and exploitation of ICTs in the Greek educational system.

The GSN project was initiated to address the primary and secondary education institutions' requirements for innovative educational practices, provide access to digital pedagogical content and establish a viable collaboration scheme between geographically distributed users' groups. Furthermore, GSN can potentially assist in defining new teaching approaches and also provide a coherent set of elearning tools that will enhance and accommodate modern digital education methodologies. Due to its innovative role the GSN fulfills the scientific and research goals for the diffusion of Internet access and the establishment and promotion of contemporary educational models and practices. Innovation is a key aspect for GSN as a means of staying on the technology frontline in the fields of information technology, network engineering and educational information systems.

GSN is also subject to academic influence since is administered by highly qualified human resources that are 
closely related to the academic community, conducting cutting edge research at related scientific areas, thus providing valuable feedback to the GSN technical community.

Through constant development and upgrade actions, GSN succeeded to maintain a thorough tested and reliable set of services, which exhibit high level of availability and scalability. The GSN services can be divided in the following categories:

- Basic services: networking provisioning, user authentication and authorization, GSN and students portal, automatic registration of users

- Communication services: email, mailing lists, instant messaging, discussion groups, etc

- Web hosting: user's page hosting, authoring tools for web pages, database service, blogs

- Advanced services: video on demand, live casting, synchronous and asynchronous e-learning teleconference, social networking

- Complementary services: school magazines, file sharing

- Central infrastructure: domain name service (DNS), lightweight directory access protocol (LDAP), distributed help desk, online statistics

- Management: users management service, network monitoring and security, remote router administration, computer emergency response team (CERT), flows collection and analysis, QoS.

Some overall statistics validating the broad use of GSN services are depicted in Table I. As is shown, there is a particularly high utilization of electronic services such as email, websites, e-Learning, social networking (educational blogs and communities) and central support (helpdesk).

TABLE I.

STATISTICAL DATA OF GSN

\begin{tabular}{|l|l|}
\hline Educational websites & $\begin{array}{l}12.747 \text { (based on PHP, Joomla, } \\
\text { Wordpress or ASP/NET technology) }\end{array}$ \\
\hline Educational blogs & 19.223 \\
\hline Educational communities & 318 \\
\hline GSN portal & $>340.000$ unique visitors per month \\
\hline Personal accounts & 84.941 (teachers), 63.554 (students) \\
\hline Active mailboxes & $>170.000$ \\
\hline Electronic courses & $\begin{array}{l}5.760 \text { from 1191 schools (current } \\
\text { school year) }\end{array}$ \\
\hline Helpdesk tickets & $>30.000 /$ year \\
\hline
\end{tabular}

GSN's flexible and well-defined architecture [3], as is also outlined from the services described, allows the potential integration of the e-learning platform described below, in terms of management, authentication and authorization and furthermore, the realization of a targeted training course appropriate adjusted to current and future technical oriented needs of computer science educators.

\section{TIME SCHEDULING AND TELE-EDUCATION PLATFORM}

A time Scheduling and Tele-Education Platform ( $t$ STEP) was designed and implemented by the Greek School Network and Networking Technologies
Directorate (http://www.cti.gr/nts) in order to provide a high quality training course that potentially compensates the growing needs of computer science educators on information and communication technologies, in both primary and secondary education. Pilot operation and detailed evaluation of the t-STEP have successfully completed, by the group of universities and research institutes that operate and maintain the GSN. Based on this valuable feedback and critical suggestions concerning the functionality of t-STEP, a production service is now available at http://training.sch.gr.

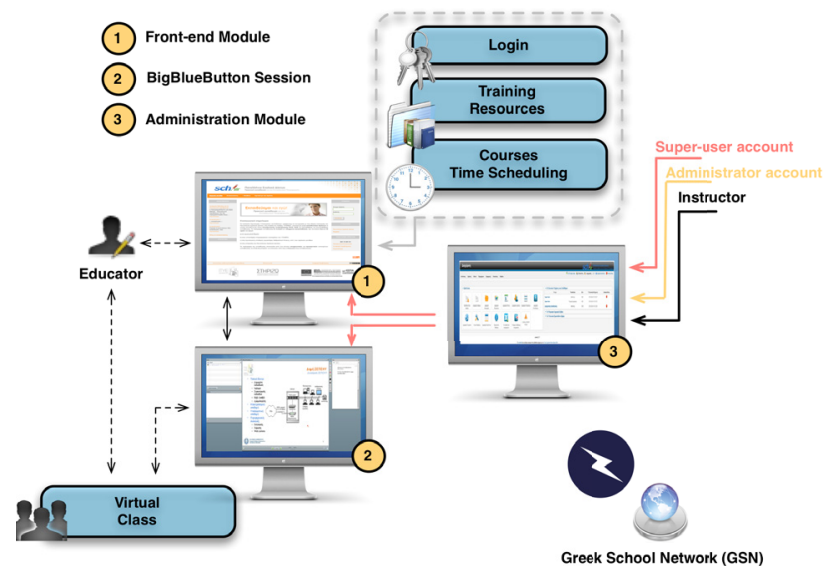

Figure 1. Conceptual overview of the e-learning platform (t-STEP)

As is depicted in Figure 1, t-STEP comprises the following components:

- Front-end module: Upon successful login (i.e., valid GSN account) time scheduling (i.e., registration in forthcoming e-lecture) and basic administration (i.e., update users' profile) operations can be efficiently performed. Also, some basic statistical data can be extracted (i.e., total hours of successfully attended e-lectures) and thoroughly presented to the user. Furthermore, a questionnaire survey is provided in order to evaluate, upon completion, the overall e-learning process, using an appropriate 5-grade scale [6]. The evaluation tasks of the electronic questionnaire are related to: (i) the functionality of the t-STEP, (ii) the instructor's technical and communication skills and (iii) the objectives and the overall organization of the educational topic. Additionally, a link to a test-room (virtual class) is provided to verify software and hardware compatibility, in order to establish the functional e-learning environment described below.

- Administration (back-end) module: The backend environment provides the main administration interface of the t-STEP, which is only accessible by the operators of the GSN and the registered instructors (with different administrative privileges). Also, a super user account (i.e., supervisor) is provided with enhanced administrative functionalities enabled. Basic operations such as monitoring the overall progress of the training course (i.e., successfully completed training sessions), scheduling or postponing a training task, tracking trainees who registered and participated in a training session, statistical data 
extraction etc., are efficiently performed through the backend module.

- Real-time e-learning environment: The realization of each e-learning session is based on the

(http://www.bigbluebutton.org/) open-source software, which is transparently integrated with the time-scheduling module. In principle, the administration of the real-time session is performed by the instructor (i.e., moderator), who can interact with the participants (i.e., mute/unmute or eject user, initiate a discussion), upload slides to the presentation area and efficiently coordinate the related learning activities (i.e., questions and discussion). The participants in each session that formulate the virtual class depicted in Figure 1 are limited to 15 .

\section{Conceptual Design of the E-LeARning COURSE}

The initial design of the training course defines 25 educational topics (ETs) organized under four categories as presented in Figure 2. The outline of the proposed training framework was based on the extensive collaboration between the administrators of the GSN [3-5] and the hints that were provided by the long-term Users Support Service (Help-Desk, http://www.sch.gr/helpdesk). Furthermore, useful information was also extracted from the ticketing system (http://helpdesk.sch.gr) utilized by the Help-Desk.

Well-documented resources designed (see Figure 3), considering fundamental adult education aspects $[7,8]$, for the realization of the proposed e-learning course and the detailed analysis and presentation of the ETs depicted in Figure 2. The training resources (see Figure 3) are available under a Creative Commons Public License (http://creativecommons.org/) and formally include (a) lecture notes complemented by practical examples and case studies, (b) instructor's presentation slides, (c) research topics and set of activities meriting further investigation and practice, (d) self-assignments (i.e., multiple choices) and (e) instructor's notes.

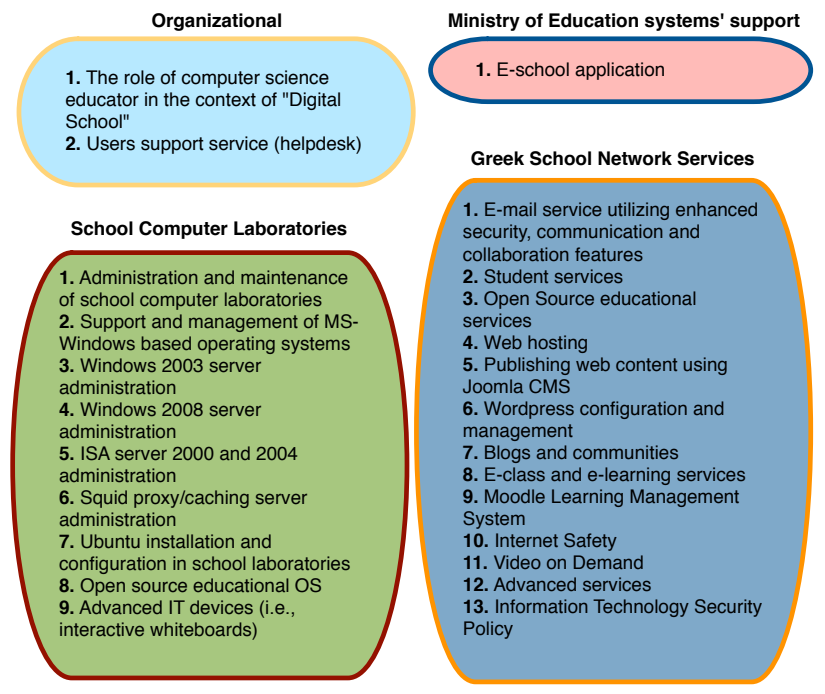

Figure 2. Educational topics of the proposed training course
As is also shown in Figure 3, a subset of the training resources (reference material) is provided to each educator. In principle, defining the appropriate mixture of availiable training resources is a challenging task. However, the motivation for designing the logical structure of the resources described above was to fullfil also the following characteristics of the proposed elearning scheme [9]:

- Address the challenges of life wide and lifelong learning

- Maximize the impact of distant learning providing in-depth and detailed training

- Assessment of e-learning and the provision of qualitative feedback (i.e., electronic questionnaire)

- Enhance an engaging and motivating learning experience and learning outcome

- Expand "educational bandwidth"

- Limit time constraints

- Establish collaborative activities and knowledgesharing

- Promote online guided discussion

- Empower self-motivation

- Perform the e-learning course as independently as possible

- Effective content and course delivery

- Potential integration with Bring Your Own Device (BYOD) methodology

- Systematically address both technical oriented tasks and pedagogical issues

- Accommodate growing students enrollment in digital technologies

- Address the growing emphasis on technological issues

- Augment central support services considering longterm support challenges

- Strengthen incentives for fostering digital technologies and encourage innovation

- Immerse computer science educators in real-world situations (i.e., school computer laboratory)

- Improve existing knowledge and technical skills

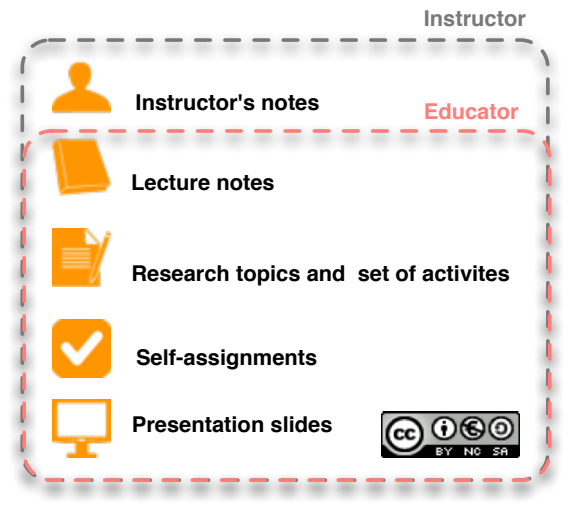

Figure 3. Reference training resources provided for the realization of the e-learning courses

The training resources, which are typical PDFs and presentation slides, are designed in a structured way in order to achieve the desired learning objectives. They can be a valuable learning resource even though they don't 
provide any interactivity and are accessible (i.e. download) through the front-end module of the t-STEP described in the previous section,

Following the conceptual framework of the intended training course, it is expected that a number of 7700 computer science educators will be served by the t-STEP, in current and forthcoming school periods (formally 20122013, 2013-2014 and 2014-2015), exploiting the robustness and functionallity of the Greek School Network. For each computer science educator a certificate of participation, that officially represents proof of training involvement, will be provided upon successful completion of ten (10) hours of e-training.

Prior to large-scale deployment of the intended elearning course, the potential interest of computer science educators to excel their technical proficiency in the aforementioned educational topics, as also as their educational needs, were examined through a preliminary electronic questionnaire survey administered from January 9th until February 3rd 2013. A total of 1469 educators participated to the questionnaire survey which corresponds to $19 \%$ of the overall computer science educators officially registered to the Ministry of Education. The evaluation of the questionnaire indicated a systematic preference for education topics related to efficient administration of school computer labs, web hosting and web content publishing using well-established CMS software, best practices for Internet safety and open source educational applications. A considerable preference for innovative educational services and advanced IT devices was also declared. On the other hand, lower preference was recorded for potential training in relative obsolete software and widely used and thoroughly tested services provided by GSN, such as the email service.

\section{Adaptation of the Proposed Training Course}

Considering the characteristics of the proposed elearning scheme and the educational topics that are currently offered (see Figure 2), it is expected that an appropriate adaptation of the provided educational topics will be performed, in order to fully exploit the outcomes of near future actions and projects, that are aiming in establishing interactive and experimental digital learning environments oriented to the Greek Educational System. This will typical include the formulation of educational topics related to the successful integration of low cost electronic platforms such as the Raspberry Pi (http://www.raspberrypi.org/) or the Arduino kit (http://www.arduino.cc/) in everyday educational and experimental practices strongly associated to programming and engineering.

Furthermore, related educational topics and activities will be developed and offered through the t-STEP, in order to efficiently support the pilot installation of lowcost $3 \mathrm{D}$ printers and potentially accelerate the large-scale adoption of this promising technology, thus strengthening the conceptual view of science and research in schools, via the processing, visualization and assessment of real products and data. In order to enhance the impact of this contemporary educational approach, a joint integration with programmable robotic and artificial intelligent systems is strongly considered as a future action in the context of the "Digital School". Thus, a complementary formulation of the training course must be performed, so that a viable support and exploitation scheme, heavily relied on the computer science educators, will be established in the long-term.

Another critical aspect is the feasible integration of the proposed e-learning framework with state of the art communication schemes, as is the promising Bring Your Own Device (BYOD) methodology that will establish a pure high-available network based education community. This will be succeeded with the broad adoption of ultraportable and smart devices, such as tablets, e-book readers, netbooks, mobile phones etc., that are simply, securely and cost-effectively connected through the existing technology infrastructure at schools. This implies that critical issues such as policy preparation, security, network capability will be thoroughly considered, thus enhancing student engagement in learning and promoting educators productivity.

\section{CONCLUSIONS}

The present work introduced a time Scheduling and Tele-Education Platform (t-STEP) exploiting the robustness of the Greek School Network (GSN) and defined the conceptual framework for a technical oriented e-learning course for computer science educators. Considering the initial design restrictions, t-STEP is tightly oriented to the Greek Educational System. Successful deployment of the scheduled e-learning course and broad acceptance from computer science educators will provide useful hints for potential use in related training and lifelong activities and also for advanced educational services for the academic community (i.e., university post-graduate courses). Given the flexible communication channels offered by hand-held devices (i.e., smartphones, tablets, netbooks etc.) the proposed elearning scheme can be potentially enhanced, although technical aspects (i.e., network resources), pedagogical issues, security and control policies, training aspects should be carefully examined. In future work, a detailed statistical analysis of the questionnaire survey feedback will be undertaken, in order to formally describe and assess the impact and the effectiveness of the training course (i.e., update and expand educational topics) complemented by a technical analysis that will investigate the potential integration with promising methodologies such as Bring Your Own Device (BYOD).

\section{ACKNOWLEDGMENT}

The authors gratefully acknowledge the contribution of Perikos Isidoros for the development of the t-STEP, Papanikolopoulou Konstantina and Stasinou Stavroula for the graphical design of t-STEP and Limperis Antonis and Aggelopoulos Panagiotis for their constructive comments and helpful suggestions.

\section{REFERENCES}

[1] J. Keyes, Bring Your Own Devices (BYOD) Survival Guide, Auerbach Publications, 2013. http://dx.doi.org/10.1201/b14050

[2] J. G. Bolanos, V. Pulkki, "Immersive Audiovisual Environment with 3D Audio Playback", 132nd AES Convention, 26-29 April 2012.

[3] M. Kalochristianakis, M. Paraskevas, E. Varvarigos, N. Xypolitos, "The Greek School Network, a paradigm of successful educational services maturing based on open source technology," IEEE 
Transactions on Education, vol. 50, issue 4, pp. 321-330, Nov. 2007. http://dx.doi.org/10.1109/TE.2007.904574

[4] M.Paraskevas, H.Stergatu, E.Varvarigos, "e-Learning and social networking services in The Greek School Network", in Proc. Int. Association for Development of the Information Society Int. Conf., Rome, Italy, pp. 105-112, July 2011.

[5] N. Xipolitos, M. Paraskevas, and E. Varvarigos, "The Greek School Network: Structure, design principles and services offered," in Proc. Int. Joint Conf. E-Business and Telecommunications, Setubal, Portugal, pp. 283-288, Aug. 2006.

[6] F. J. Fowler, Improving Survey Questions: Design and Evaluation, Sage Publications, 1995.

[7] L. Cohen, L. Manion, K. Morrison, Research Methods in Education, Taylor \& Francis Group, 2011.

[8] W. Horton, Designing Web-Based Training, John Wiley, 2000.

[9] L. M. Harasim, S. R. Hiltz, L. Teles, M. Turoff, Learning Networks: A Field Guide to Teaching and Learning On-Line, MIT Press, 2001

\section{AUTHORS}

M. Paraskevas received an electrical engineering degree in 1989 from the University of Patras, Greece. In 1995 he received the $\mathrm{PhD}$ degree in digital signal processing area. In 2011 he became Assistant Professor at Informatics Engineering Department of Technological Educational Institute of Western Greece. He is also the Director of the Directorate of Greek School Network (www.cti.gr/nts) at Computer Technology Institute and Press "Diophantus" (www.cti.gr/en), which through its involvement in pioneering research and development projects, has a major role in the development of network technologies and telematic services in Greece. His current research interests are in signal theory and DSP, analogue and digital communications, next generation networks, egovernment and e-learning services, as well as in the exploiting the ICTs in the education. He has published more than 30 scientific articles in international magazines and conferences and also he has participated in scientific committees of international journals and conferences. (email: mparask@cti.gr)

T. Zarouchas received the Diploma of Electrical and Computer Engineering in 2002 and the Ph.D. degree in Electrical and Computer Engineering in 2010, both from University of Patras, Greece. His main research interests are in the area of digital signal processing for audio applications, perceptual audio coding, psychoacoustics, audio quality evaluation and room acoustics, applications for digital audio and multimedia communication networks and wireless technologies oriented to multimedia applications. He has authored and presented his research work in scientific journals and international conferences and has co-authored two textbooks for the postgraduate course "Acoustic Design - Multimedia" of the Hellenic Open University. Since 2010 collaborates with the Technological Educational Institute (TEI) of Patras as an adjoint assistant professor in the Department of Informatics \& Mass Media. In 2012 he joined the Greek School Network and Networking Technologies Directorate at the Computer Technology Institute and Press "Diophantus". Dr. Zarouchas is a member of the Institute of Electrical and Electronics Engineers (IEEE), the Technical Chamber of Greece and the Hellenic Institute of Acoustics. (e-mail: zarouchas@cti.gr)

The related activities that led to these results were co-funded by National Funds and by the European Social Fund (ESF), under the Hellenic National Strategic Reference Framework (NSRF) 2007-2013. Manuscript received 15 May 2013. Published as resubmitted by the authors 23 July 2013. 